\title{
Ultra-Short-Term wind speed prediction using RBF Neural Network
}

CAO Gao-cheng ${ }^{1,3, \text { a }}$, HUANG Dao-huo ${ }^{2, b}$

${ }^{1}$ Electric Power Occupational Technical Institute of SEPC,Taiyuan 030021,China;

${ }^{2}$ Huadian Electric Power Research Institure,Hangzhou 310000, China

${ }^{3}$ Hebei Engineering Research Center of Simulation \& Optimized Control for Power Generation,

North China Electric Power University, Baoding 071003, China

agaocheng_cao@163.com, bdaohuo-huang@chder.com

\begin{abstract}
As a renewable and clean energy source, wind power is being widely utilized all over the world. The uncertainty of wind speed makes certain trouble for the development of wind power generation. In order to relieve the disadvantageous impact of wind speed intermittence on connected power system, this paper proposes a radial basis function (RBF) neural network-based prediction model for ultra-short-term wind speed. Simulation studies are carried out to validate the proposed model for ultra-short-term wind speed by using data obtained from a wind farm from Beijing. The performance of the RBF neural network is compared with that of BP network. Results show that the RBF prediction model significantly outperforms the BP model.
\end{abstract}

Keywords: wind speed; radial basis function (RBF); ultra-short-time prediction

\section{Introduction}

The development and utilization of renewable energy, especially wind energy has been attached great importance all over the world. The rapid growth of wind power installed capacity will affect the safety and stability operation of the power grid, and power prediction is one of the effective ways to solve the problem. 
Artificial Neural Network (ANN) can extract and approximate nonlinear relationship between the input and output through learning process. Therefore, the methods based on artificial neural network of wind speed and wind farm output prediction have become one of research hot spots. At present, the commonly used artificial neural networks mainly contain BP neural network, the local feedback neural network and so on. Although the results in the overall trend are in agreement with the measured values, but the numerical errors are still relatively large. When BP neural network function is applied in approximation, negative gradient descent method is used to adjust weights. The result indicates slow convergence and is easy to fall into local minima. Researches [1-3] show that RBF neural network has advantages such as high approximation precision, nonexistence of local minimum problem. And in the field of approximation capability, analysis ability and learning speed, RBF neural network is significantly superior to other artificial neural network.

Therefore, a wind speed-forecasting model is established based on RBF neural network and combined with the measured data of a wind farm to verify the accuracy and reliability of the model. Compared with the BP neural network forecasting model, it can be concluded that RBF model is improved in precision and reliability than BP prediction model.

\section{RBF neural network algorithm and prediction system}

\section{A. RBF neural network}

Artificial neural network is a large-scale parallel distributed and nonlinear information processing system[4]. RBF neural network, as showing in Fig.1, has three layers. The input layer is simply a set of sensory units. The second layer is a hidden layer of sufficient dimension, which performs a nonlinear transformation from the input space to a higher-dimensional hidden-unit space. Its action function (basis function) is usually Gauss Function. When the input information is close to the central area of the basic function, the hidden layer nodes will have a greater output. Thus, this network has a good capacity of local approximation, as well as fast learning speed. The third layer performs a linear transformation from the 
hidden unit space to the output space. In case that there are enough hidden nodes, it could approximate any nonlinear function[5].

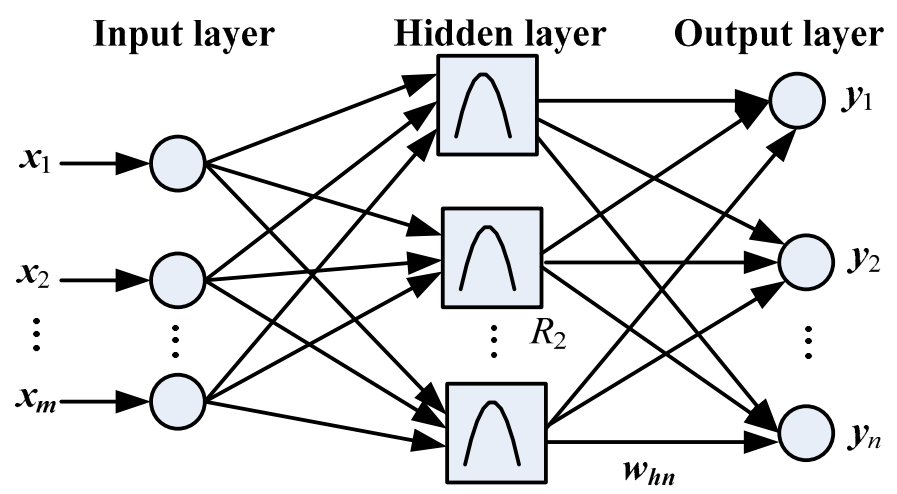

Fig.1 RBF neural network structure

\section{B. RBF network learning algorithm}

There are three parameters to be solved in RBF neural network learning algorithm, namely the center of the basis function, the variance and weights from hidden layer to output layer[6]. Gauss Function is usually used as the basis function of RBF network. Therefore the active function can be expressed as Eq.1:

$$
R\left(x_{p}-c_{i}\right)=\exp \left(-\frac{1}{2 \sigma^{2}}\left\|x_{p}-c_{i}\right\|^{2}\right)
$$

Where $\left\|x_{p}-c_{i}\right\|$ is the European norm, $c$ is the center of Gauss function, $\sigma$ is the variance of Gauss function.

The output of network can be obtained from the RBF neural network structure which is shown as Eq.2:

$$
y_{j}=\sum_{i=1}^{h} w_{i j} \exp \left(-\frac{1}{2 \sigma^{2}}\left\|x_{p}-c_{i}\right\|^{2}\right) j=1,2, \cdots, n
$$

Where $\quad x_{p}=\left(x_{1}^{p}, x_{2}^{p}, \cdots, x_{m}^{p}\right)^{T} \quad$ is the $\quad p$-th input sample, $p=1,2, \ldots, \quad P$ represents the total number of samples; $c_{i}$ is the center of network hidden layer nodes; $w_{i j}$ is the connection weight from hidden layer to output layer; $i=1,2, \cdots, h, h$ is the number of hidden layer nodes; $y_{j}$ is the actual output of the $j$-th network output node corresponding with the input sample[7]. 
Suppose $d$ is the expected output of sample, then the variance of basis function can be expressed as Eq.3:

$$
\sigma=\frac{1}{P} \sum_{j}^{m}\left\|d_{j}-y_{j} c_{i}\right\|^{2}
$$

\section{Prediction Evaluation Standard}

To evaluate the accuracy of RBF approach in forecasting wind speed, different criterions are used. The relative percentage error (RPE) criterion, the mean absolute percentage error (MAPE) criterion are defined as follows.

$$
\begin{gathered}
M A P E=\frac{1}{N} \sum_{i=1}^{n}\left|\frac{V_{i}-\hat{V}_{i}}{V_{i}}\right| \times 100 \% \\
R P E=\left|\frac{V_{i}-\hat{V}_{i}}{V_{i}}\right| \times 100 \%
\end{gathered}
$$

\section{The RBF neural network applied to wind speed}

Using an asynchronous wind power generator whose capacity is $1.5 \mathrm{MW}$ from a wind farm in Beijing as an example, this paper forecasts the wind speed for 1 minute in advance by RBF neural network method using the measured wind speed and power data per minute in 2010 June 1-4. The number of learning sample is 4260 , the testing sample is 30 and the time interval is 1 minute.

For convenience, $v(k)$ represents the wind speed in the current time, $v(k-1)$ represents the past 1 moment wind speed, $v(k-2)$ represents the past 2 moments wind speed, $v(k+1)$ represents the wind speed of 1 moment in advance.

When using the following variables as the wind speed prediction model inputs, the overall error of wind speed prediction reaches the minimum. Therefore the basic structure of RBF neural network prediction models for wind speed is shown as Fig. 2: 


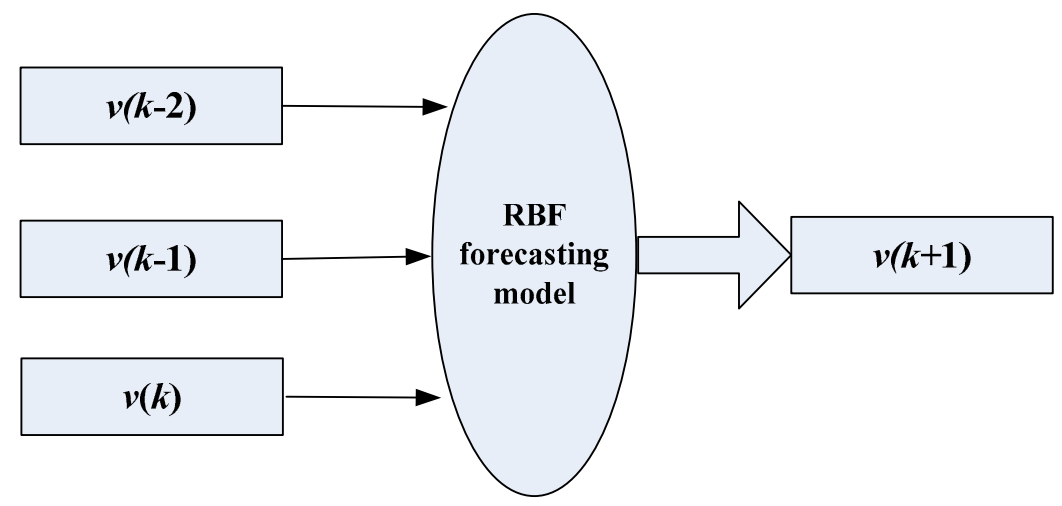

Fig. 2 Structure of RBF neural network prediction models for wind speed of $1 \mathrm{~min}$ in advance

In the RBF network learning process, when the data is far from the null range, learning speed is slow. In order to improve the training and forecasting speed, the wind speed in the dataset data should be normalized, and the data is mapped to the range from -1 to 1 . In the end of training, the simulation output will be anti-normalized. Normalized function is shown as Eq.6:

$$
v^{\prime}=\frac{2\left(v-v_{\min }\right)}{v_{\max }-v_{\min }}-1
$$

Where $v^{\prime}$ is normalized data, $v$ is original data, $v_{\min }$ is minimum of original data, $v_{\text {max }}$ is maximum of original data.

The function of newrbe() from MATLAB toolbox is used to construct RBF function networks and make the design error meet zero. The function is called as: net=newrbe ( $\mathrm{P}, \mathrm{T}$, spread), where $\mathrm{P}$ is the input vector and $\mathrm{T}$ is desired output vector (goal) and spread is the distribution density of RBF. And in RBF spread is the only parameter that needs to be determined. It's very important to select a reasonable size for spread. With the value of spread increasing, it becomes much easier for RBF to respond the interval covered by input vector and the output is much smoother. But it will result in the great difficulty in numerical calculation. When the size of spread is 51 , the training sample error gets the relatively excellent results by experiments.

Fig. 3 compares the prediction results of using the RBF neural network with BP neural network. The BP network is divided into three layers. The number of 
input layer neurons is equal to the number of input variables (namely 3 inputs)and the number of hidden layer neurons is 18 . The transfer function of hidden layer neurons is S-tangent function and linear function is used as the transfer function of the output layer neurons.And the Levenberg-Marquardt back-propagation algorithm is used as the BP neural network training function. The learning rate of BP networks is set as 0.01 and training target goal is 0.01 .

The RPE figure shown as Fig.4 can be obtained from the experiment which indicates the $1 \mathrm{~min}$ ahead prediction of wind speed of continuously $30 \mathrm{~min}$ data based on the above formula.From the figure,we can get that the RPE maximum of $\mathrm{RBF}$ is $11.99 \%$ and MAPE is $6.9 \%$, while the RPE maximum of BP is $23.60 \%$ and MAPE is $10.7 \%$.

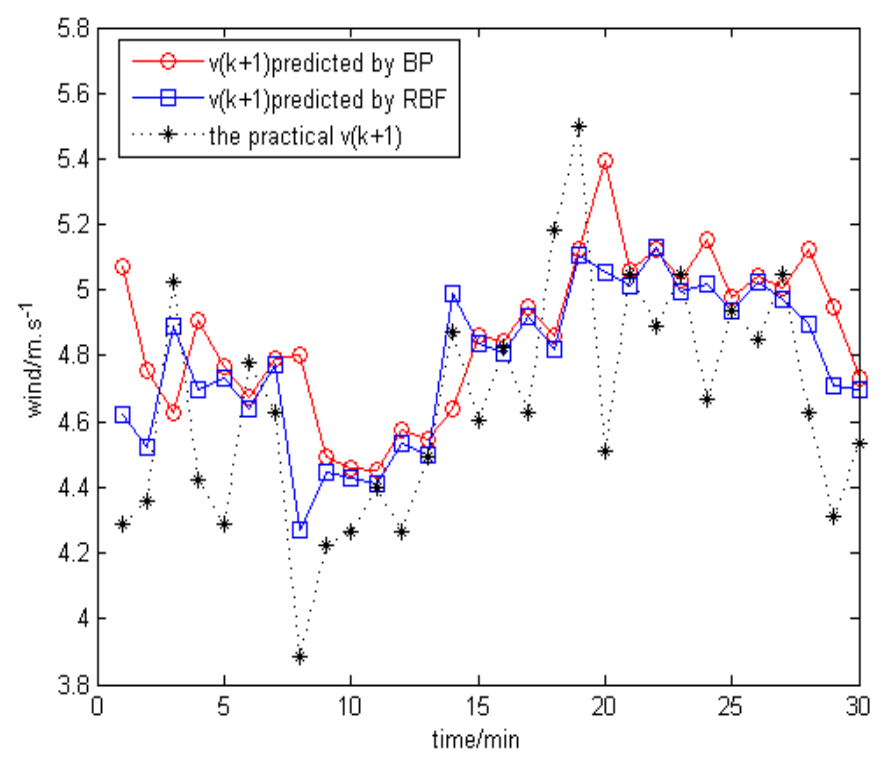

Fig. 3 the predicted results of $v(k+1)$ 


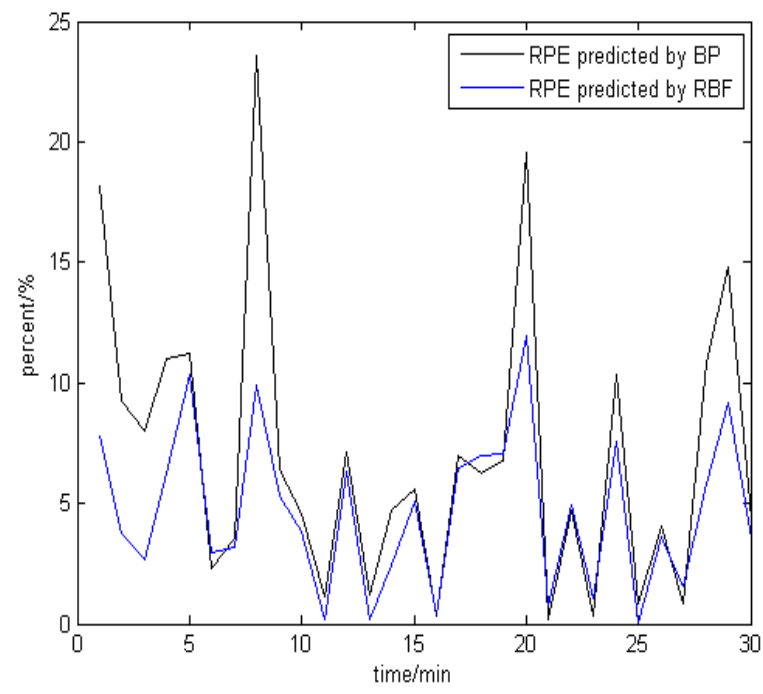

Fig. 4 RPE of RBF and BP methods

\section{Summary}

This paper has presented a RBF neural network-based prediction model for wind speed, which has been compared with BP prediction model. Simulation studies using the data from a wind farm in Beijing have yielded several conclusions. First, the proposed RBF neural network-based prediction model has better performance than the BP prediction model in terms of the prediction accuracy. This is due to the RBF neural network's ability of capturing nonlinear and time-varying nature of the wind speed data. Second, simulation results have indicated the success of using historical wind speed variables to improve the prediction accuracy.In future work, other nonlinear prediction models will be compared with the RBF neural network-based model.

\section{References}

[1] Liu Zhigang, Wang Xiaoru, He Zhengyou, et al. Analysis andcomparison of function approximation ability based on wavelet transformation, neural network 
and wavelet network[J] .Automation of Electric Power Systems, 2002, 26(20) : 39-44(in Chinese).

[2] Lei Shaolan, Sun Caixin, Zhou Quan, Zhang Xiaoxing, Cheng Qiyun.Short-term load forecasting method based on RBF neural network and anfis system[J]. Proceedings of the CSEE, 2005, 25 (22) : 78-82.

[3] XiaoYongshan, Wang Weiqing,Huo Xiaoping. Study on the Time-series Wind speed forecasting of the wind farm based on neural networks. Energy conservationtechnology, 2007, 25(2)106-108.

[4] Huang Xiaohua, Li Deyuan, Lv Wenge. Wind speed prediction based on artificial neural network model[J]. Acta energiae solaris sinica, 2011, 32(2): 193-197(in Chinese).

[5] Liu Jinbao, Ding Tao. Wind speed prediction based on RBF neural network[J]. Acta energiae solaris sinica, 2012, 33(7): 1131-1135(in Chinese).

[6]Yang Xiuyuan, Xiao Yang, Chen Shuyong. Wind speed and generated power forecasting in wind farm[J]. Proceedings of the CSEE, 2005, 25(11): 1-5. [7]Mu Yuqiang, Wang Xiuli, Bie Zhaohong, et al. Analysis of wind speed probability distribution and wind turbine generator capacity factor[J]. Power System protection and control, 2009, 37(1): 65-70. 